\title{
Development of a Low Temperature PZT/Polymer Paste for Screen Printed Flexible Electronics Applications
}

\author{
Ahmed Almusallam, Kai Yang, Dibin Zhu, Russel Torah, John Tudor and Steve Beeby \\ Electronics and Computer Science, University of Southampton, Southampton, UK \\ Email: asa1g09@ecs.soton.ac.uk
}

\begin{abstract}
This Screen-printing is a versatile and cost effective fabrication method for depositing thick-film patterns on a wide range of substrates. It has been used in printing electrics on flexible substrates such as films papers and textiles. This paper presents a low temperature screen-printable PZT/polymer paste consisting of PZT powder, high dielectric constant polymer binder and solvent. The optimization of the PZT/polymer weight ratio was investigated in order to achieve a high $d_{33}$ of $40 \mathrm{pC} / \mathrm{N}$ has been obtained after poling the device using an optimized process of $90{ }^{\circ} \mathrm{C}$ for 6 minutes with an applied electric field of 3.75 MV/m. Also, the material has a Young's modulus of 131 MPa and flexible to be exploited in smart-fabric applications for energy harvesting and sensing.
\end{abstract}

Keywords-Piezoelectric; flexible electronics; energy harvesing; sensing

\section{INTRODUCTION}

Piezoelectric materials show a great attention from researchers to be exploited in sensors and energy harvesting. The relation between polarization and applied mechanical stress could be well-employed in sensors and energy harvesters (direct effect). However, the relation between mechanical strain on the piezo-element and applied electrical voltage can be used in actuators (inverse effect) [1].

Such smart materials that can be used in sensors, energy harvesters and actuators can be well-exploited in smart-fabrics and flexible electronics applications. Using piezoelectric materials in smart-fabrics and flexible electronics applications requires the piezoelectric material to be flexible and processed at lower temperatures. Also, using materials in screen-printing can increase the mass-production of the proposed devices as it is a forthright fabrication method.

Only piezoelectric composites can be adopted and used in screen-printing. The piezoelectric polymer (PVDF) and its copolymers (e.g. PVDF-TrFE) are not adaptable to screenprinting and also require some pre-poling procedures (e.g. stretching) to be properly polled. Most of the proposed piezoelectric composites are biphasic materials (i.e. a mixture between piezoelectric ceramic and a polymer). The polymer can be piezoelectric or non-piezoelectric polymer. Mixing piezoelectric polymers with piezoelectric ceramic has a negative effect on the overall piezoelectric properties (i.e. $d_{33}$ ) of the composite [2]. Lots of contributions was achieved in producing piezoelectric composites [2-4] and only concerned about piezoelectric activity but none of them were concerned about mechanical flexibility and the effect of polymer binder polarity on the piezoelectric activity of the printed film.

The piezoelectric polymers exhibits opposite polarization to their ceramic counterparts when simultaneously poled with same direction external electric field. This opposite polarization can reduce the overall $d_{33}$ of the piezoelectric composite by cancelling the polarization of each piezoelectric phase. Mixing piezo-ceramic powder material with nonpiezoelectric polymer with high polarity polymer (i.e. 0-3 connectivity) can be a solution for this problem. This type of connectivity was used because it is easy for mixing, compatibility of screen-printing and help increases mass production.

This paper offers a new screen-printed, low temperature biphasic composite with 0-3 connectivity. The composite shows great flexibility to be printed on flexible mediums such as fabrics. The composite show greater piezoelectric and dielectric properties compared to our previous work [5, 6]. These properties are explored and discussed in this paper.

\section{EXPERIMENTAL}

\section{A. Mixing Formulations}

The PZT-5H ceramic powder (Pz29, Ferroperm, Piezoceramic) used in this study was a mixture of $2 \mu \mathrm{m}$ and 0.8 $\mu$ particles with a weight ratio of $4: 1$. This PZT powder was blended with the high polarity polymer. As the dielectric and piezoelectric properties of the material are investigated in terms of PZT weight loading inside the polymer matrix, the PZTpolymer weight ratios were varied. The four investigating PZTpolymer weight ratios were 8:1, 10:1, 12:1 and 14:1.

The PZT ceramic and the polymer were mixed with these ratios resulting four screen-printed pastes. Triple roll mill was used to homogenously spread the PZT particles inside the polymer matrix. This will lead to a good quality screen-printed paste without lumps and blocking the mesh openings of the screen during printing. Also, homogenously dispersing the PZT particles will lead a printed film with good mechanical and electrical properties. 


\section{B. Screen-printing the Blended Pastes}

The four formulations were separately screen-printed using a DEK 248 screen-printer on Kapton polyimide $300 \mathrm{HN}$ between screen-printed electrodes creating a capacitor structure shown in Fig.1. The Kapton substrate has a thickness of $75 \mu \mathrm{m}$.
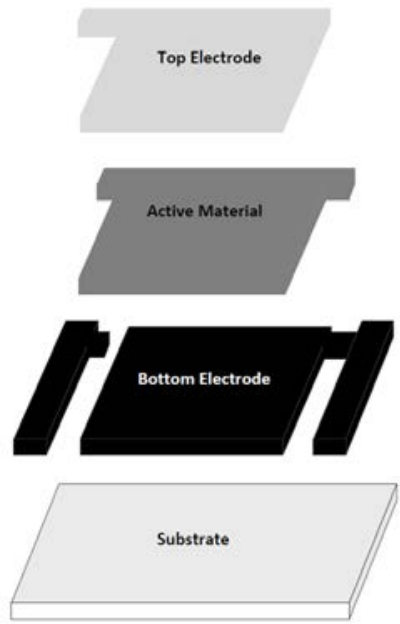

(a)

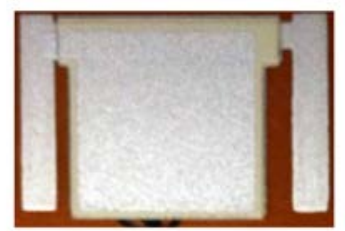

(b)

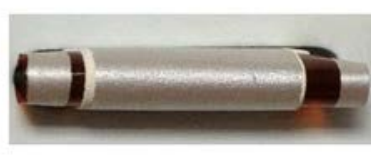

(c)
Fig.1. Capacitive test structure. Schematic of test capacitive structure of the film (a). Actual printed device (b) and showing the flexibility of the printed films by a complete bending

Low temperature and screen-printed silver-polymer paste (DuPont 5000) was used as bottom and top electrodes. As PZT powder was thermally processed at higher temperature compared to the polymer matrix, therefore curing temperature of the formulations will be dominated by the polymer curing conditions. The printed films have been dried and cured in a box oven at curing conditions shown in table 1 which are appropriate for smart-fabric applications. Although the bottom and top electrode are from the same type, the top electrode was cured in a low temperature and time so that it does not affect the piezoelectric composite and over cure it which might lead to a change in the properties of the polymer and therefore the whole printed film.

TABLE I. THE CURING CONDITIONS OF THE PRINTED FILMS

\begin{tabular}{|l|c|c|}
\hline \multicolumn{1}{|c|}{ Material } & $\begin{array}{c}\text { Curing temperature } \\
\left({ }^{\circ} \mathbf{C}\right)\end{array}$ & $\begin{array}{c}\text { Curing time } \\
(\text { min) }\end{array}$ \\
\hline $\begin{array}{l}\text { Bottom electrode } \\
(\text { DuPont 5000) }\end{array}$ & 120 & 10 \\
\hline PZT/polymer & 90 & 6 \\
\hline $\begin{array}{l}\text { Top electrode } \\
\text { (DuPont 5000) }\end{array}$ & 90 & 6 \\
\hline
\end{tabular}

\section{Poling the Piezoelectric Devices}

Poling piezoelectric film is an essential stage to activate the piezoelectric material. The devices were polled by applying an external electric field simultaneously with elevating temperatures for a specific time. Applying electric field causes the dipoles inside the piezoelectric particles to be aligned. The elevating temperatures provide the energy required to facilitate and help easily realign the dipoles.

There are several poling methods but direct contact poling was used for easy-setup and decent spreading of electric field across the piezoelectric material according to the effective area (i.e. area of the electrodes). The applied electric field can be estimated according to the following equation $\mathrm{E}=\mathrm{V} / \mathrm{d}$, where $\mathrm{E}$, $\mathrm{V}$ and $\mathrm{d}$ are the external electric field, applied voltage and thickness of the screen-printed piezoelectric material, respectively.

\section{RESUlTS AND DESCUSSIONS}

\section{A. Porosity and Dielectric Porperties}

Good dielectric properties (i.e. can be described as dielectric constant) of the piezoelectric material lead to a good piezoelectric properties of the film. The remnant polarization can be improved if the dielectric properties are high. This is because higher dielectric constant will lead the effective electric field on the PZT particles is higher and consequently the repentant polarization increases. The higher the remnant polarization of a piezoelectric material, the higher the piezoelectric properties of the material will be. Choosing the proper polymer to be mixed with the PZT particles is important as it affects the overall dielectric properties of the composite.

Table 2 shows the dielectric constants of the two composite constituents provided from the manufacturers. However, when both of them are mixed, the total dielectric constants will be averaged including the air voids which will lower down the overall dielectric constant of the composite.

TABLE II. DIELECTRIC PROPERTIES OF THE TWO CONSTITUENTS

\begin{tabular}{|c|c|}
\hline Material & Dielectric constant \\
\hline PZT-5H & 2900 \\
\hline Polymer & 15 \\
\hline
\end{tabular}

The actual dielectric constants of the PZT/polymer with different PZT loading are shown in Fig.1. The dielectric constant increased up to 179 from PZT/polymer weight ratio of 8:1 to $10: 1$. However, this value is decreased at higher PZT powder weight loading due to the increase of the air voids inside the composite. At a certain point of PZT loading, the air voids increases and the dielectric constant is dropped down. When the number of PZT particles is increased on the account of the polymer, the polymer quantity is reduced and some gaps and voids are left to be filled with air. The increase of PZT powder inside the polymer matrix at a certain limit (threshold point quantity) increase the air voids inside the composite and the dielectric properties is reduced. Investigation in PZT powder loading is required to know about this threshold point, otherwise the increase of the PZT powder will not be useful and might affect negatively the dielectric and piezoelectric properties. 


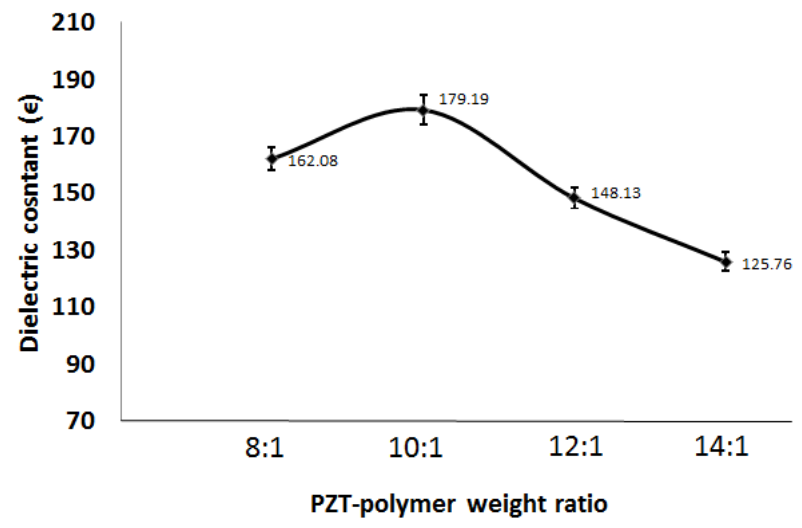

Fig.2. Dielectric constants of different PZT weight loading inside the polymer matrix

However, most of piezoelectric composites that are mixed with $0-3$ connectivity suffer from porosity and air voids within the material as shown in Fig.3. These air voids affect the dielectric and therefore the piezoelectric properties of the printed film. Also, air voids can change the expected mechanical properties (e.g. reduction in Young's modulus).

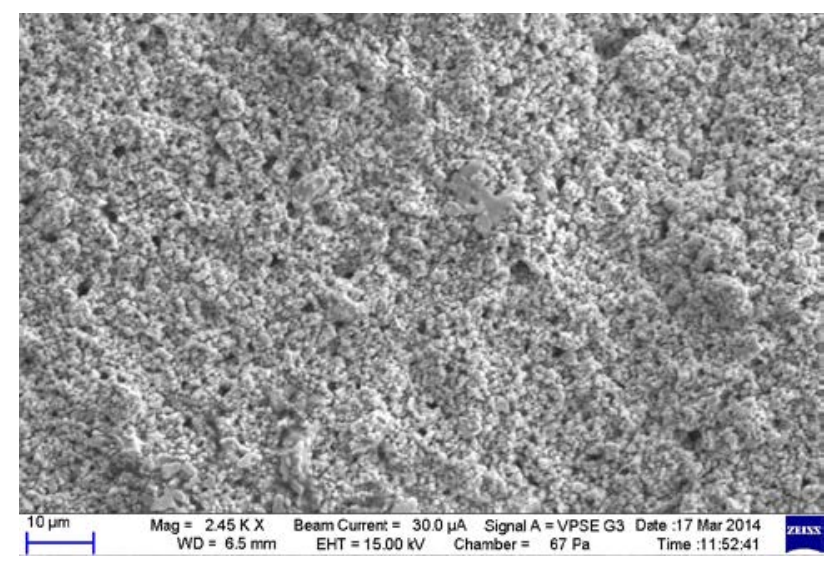

Fig.3. SEM image of the PZT/polymer showing the PZT particles spreading in the polymer matrix of 12:1 PZTpolymer weight ratio

\section{B. Intial $d_{33}$ measurements of the mateirals}

The piezoelectric properties $d_{33}$ were investigated over different $\mathrm{PZT} /$ polymer weight ratios. The poling conditions $\left(\mathrm{E}=2 \mathrm{MV} / \mathrm{m}, \mathrm{T}=100{ }^{\circ} \mathrm{C}\right.$ and $\left.\mathrm{t}=4 \mathrm{~min}\right)$ were applied the same to all the $\mathrm{PZT} /$ polymer weight ratios devices. Four devices were used for every weight ratio and 5 measurements were taken for each device. The total $d_{33}$ measurements for every weight ratio were 20 . The mean and standard deviation values of the $d_{33}$ measurements for PZT/polymer weight ratios are shown in Fig.4.

Fig.4 shows an increase in $d_{33}$ from weight ratios 8:1 up to $12: 1$. However, the $d_{33}$ is reduced after this ratio due to the effect of air voids and dielectric constant. PZT/polymer weight ratios of $10: 1$ and $12: 1$ showed good $d_{33}$ measurements of 32.2 and $33.7 \mathrm{pC} / \mathrm{N}$, respectively.

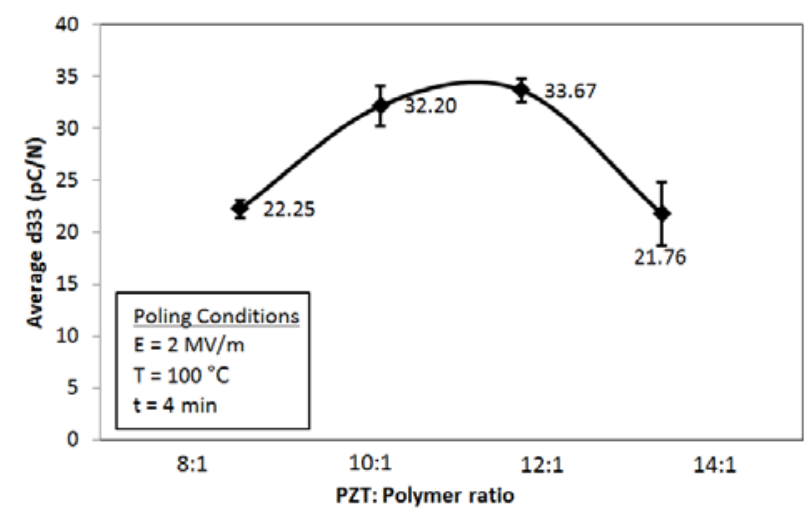

Fig.4. $d_{33}$ measurements of the poled devices on Kapton

\section{Mechanical Properties of the Selected Material}

The mechanical properties were measured for the material that gives the highest piezoelectric properties with $\mathrm{PZT} /$ polymer weight ratio of $12: 1$. It was this PZT/polymer ratio gives the highest piezoelectric properties and good mechanical flexibility as shown in Fig.1c. The Young's modulus of the PZT/polymer material with weight ratio of $12: 1$ is $131 \mathrm{MPa}$.

\section{Optimising Poling Conditions}

In order to obtain maximum remnant polarization and therefore maximum piezoelectric properties $\mathrm{d}_{33}$, poling conditions which are represented by electric field (E), temperature $(\mathrm{T})$ and time $(\mathrm{t})$. The poling conditions for 12:1 PZT/polymer composite material were optimized. Poling temperature was fist investigated with initial poling conditions $\mathrm{E}=2 \mathrm{MV} / \mathrm{m}$ and $\mathrm{t}=4 \mathrm{~min}$. Four devices were employed for poling condition optimization and five measurements have been taken from each point. Fig. 5 shows increasing poling temperature, increases $d_{33}$ from 80 to $90{ }^{\circ} \mathrm{C}$. However, the $d_{33}$ was reduced after $90{ }^{\circ} \mathrm{C}$ due to the sensitivity of the polymer inside the composite to temperatures above curing.

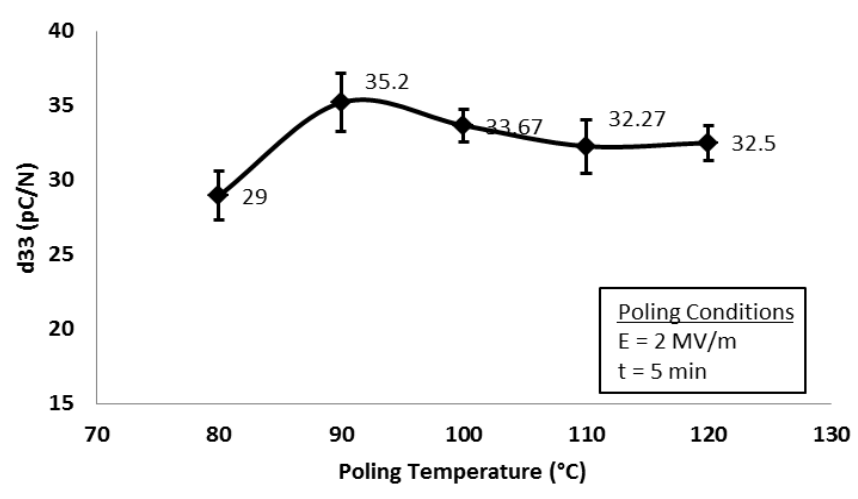

Fig.5. Optimizing poling temperature

Poling time was then explored with the following poling conditions $\left(\mathrm{E}=2 \mathrm{MV} / \mathrm{m}\right.$ and $\left.90{ }^{\circ} \mathrm{C}\right)$. The optimum poling 
temperature was used because all poling parameters are interrelated. It was found from the results in Fig.6 that the optimum poling time is 6 min gives a $\mathrm{d}_{33}$ of $38.7 \mathrm{pC} / \mathrm{N}$.

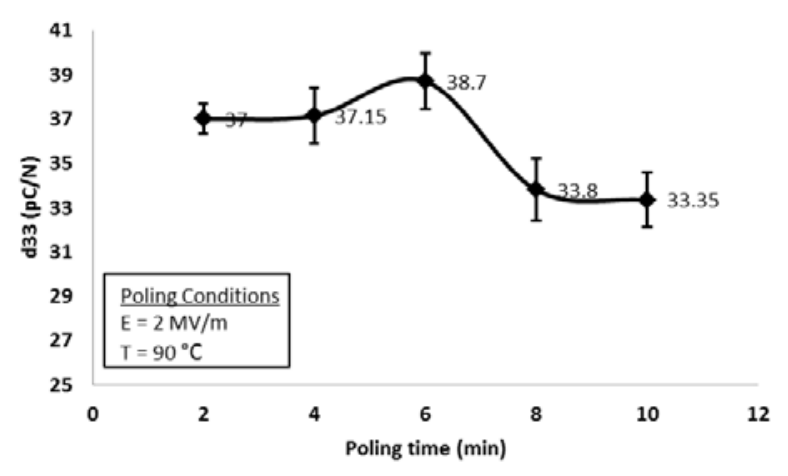

Fig.6. Optimizing poling time

Finally, $\mathrm{d}_{33}$ values against poling electric field is investigated with other optimum poling conditions are fixed ( $\mathrm{T}=90{ }^{\circ} \mathrm{C}$ and $\mathrm{t}=6 \mathrm{~min}$ ). Best electric field that gives the highest $\mathrm{d}_{33}$ value was $3.75 \mathrm{MV} / \mathrm{m}$. Beyond this field (e.g. at 4 $\mathrm{MV} / \mathrm{m}$ ) the electric $d_{33}$ is reduced to $36.73 \mathrm{pC} / \mathrm{N}$. This is due to the instability in poling because of the trip current passing across the piezoelectric element. This current was caused due to the reduction in the overall resistivity of the film due to the air voids inside. This problem can be solved by isostatic pressing the material pushing the air inside the film out and filling it with the main composite material.

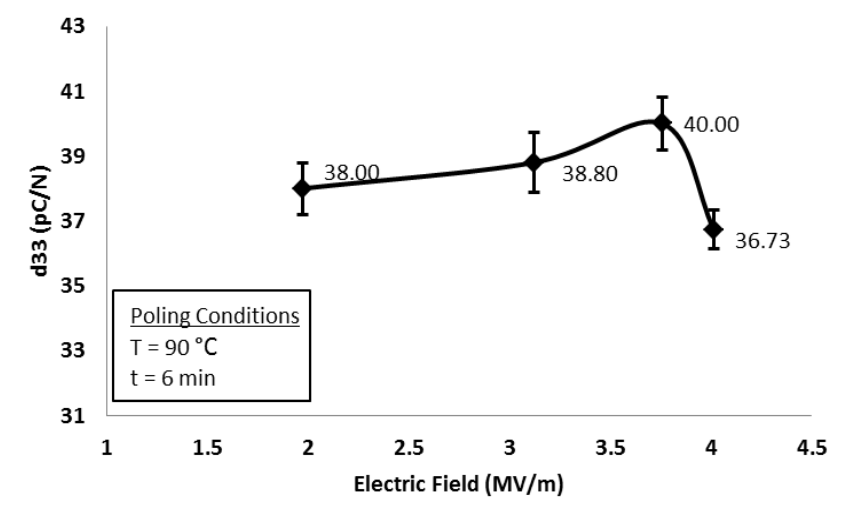

Fig.7: Optimizing poling electric field

\section{CONCLUSTION}

A high polarity polymer was employed to produce a new flexible, low temperature and screen printable PZT/polymer composite with 0-3 connectivity, which can be used in energy harvesting and sensing particularly in flexible electronics applications (e.g. smart fabrics). Four PZT powder weight loading was varied inside in the polymer matrix and dielectric and piezoelectric properties were investigated.
Amongst the four formulations, 12:1 PZT/polymer weigh ratio has been recognized giving a $d_{33}$ of $33.67 \mathrm{pC} / \mathrm{N}$. However, 10:1 PZT/polymer provides the highest dielectric constant of 179. After optimizing the poling condition for 12:1 PZT/polymer material, the $d_{33}$ values increased to $40 \mathrm{pC} / \mathrm{N}$. This material is apposite for use on smart-fabric applications whether in energy harvesting as it gives high $\mathrm{d}_{33}$ values compared to PVDF and in also in sensing.

\section{REFERENCES}

[1] E. Venkatragavaraj, B. Satish P.R. Vinod and M.S. Vijaya, "Piezoelectric properties of ferroelectric PZT-polymer composites," Journal of Physics D: Applied Physics, vol. 34, pp. 487-492, 2001.

[2] M. Dietze and M. Es-Souni., "Structural and functional properties of screen-printed PZT-PVDFTrFE composites," Sensors and Actuators, vol. 143, pp. 329-334, 2008.

[3] T. Papakostas and N.M. White, "Screen printable polymer piezoelectrics, ” Sensor Review, vol. 20, pp. 135 - 138, 2000.

[4] Y.H. Son, S.Y. Kweon, S.J. Kim, Y.M. Kim, T.W. Hong and Y.G. Lee, "Fabrication and electrical properties of PZT-PVDF 0-3 type composite film," Integrated Ferroelectrics, vol. 88, pp. 44-50, 2007.

[5] A. Almusallam, R.N. Torah, K. Yang, J. Tudor and S.P. Beeby, "Flexible Low Temperature Piezoelectric Films for Harvesting from Textiles,” presented at the PowerMEMS 2010, December 3-6, Atalanta, USA, Atalanta, USA, 2012.

[6] A. Almusallam, R.N. Torah, D. Zhu, M.J. Tudor, and S.P. Beeby, "Screen-printed piezoelectric shoeinsole energy harvester using an improved flexible PZT-polymer composites," presented at the PowerMEMS 2013, London, 2013. 